Editor's Note: These short reviews of a recent paper in the Journal, written exclusively by graduate students or postdoctoral fellows, are intended to mimic the journal clubs that exist in your own departments or institutions. For more information on the format and purpose of the Journal Club, please see http://www.jneurosci.org/misc/ifa_features.shtml.

\title{
Acquiring Visual Object Expertise: Reorganization in the Ventral Path
}

\author{
Kai-Markus Müller \\ Unit on Cognitive Neurophysiology and Imaging, National Institute of Mental Health, Bethesda, Maryland 20892, and International Max-Planck Research \\ School, Graduate School of Neural and Behavioural Sciences, 72074 Tübingen, Germany \\ Review of Op de Beeck et al. (http://www.jneurosci.org/cgi/content/full/26/50/13025)
}

Evolution has allocated the largest portion of the primate sensory brain for visual processing. This may reflect the fact that even seemingly trivial visual problems, such as discriminating between two shapes, require exquisitely tuned perceptual machinery. The so-called ventral path is crucial for discriminating, storing, and recognizing visual objects, which includes areas from the occipital to the temporal lobe (Logothetis and Sheinberg, 1996). Like many others skills, visual discrimination can improve through training. However, it is not clear how this is accomplished for particular visual patterns, such as when a repeatedly seen stimulus set becomes more familiar and objects within that set are better discriminated.

A controlled approach to investigating how the brain acquires visual expertise is to create parameterized fantasy objects and train subjects to discriminate or categorize them. For example, Gauthier et al. (1999) asked subjects to study so-called "Greebles," a set of objects sharing common spatial configurations. This study showed that in functional magnetic reso-

Received Feb. 13, 2007; revised March 20, 2007; accepted March 21, 2007.

This work was supported by The Division of Intramural Research Programs at the National Institute of Mental Health. I thank Dr. David A. Leopold, Dr. Alexander V. Maier, Cynthia J. Chang, and the reviewing editor for valuable comments.

Correspondence should be addressed to Kai-Markus Müller, Unit on Cognitive Neurophysiology and Imaging, National Institute of Mental Health, 49 Convent Drive, Room B2J-45, Mail Stop Code-4400, Bethesda, MD 20892. E-mail: muellerkm@mail.nih.gov.

D0I:10.1523/JNEUROSCI.0634-07.2007

Copyright $\odot 2007$ Society for Neuroscience $\quad$ 0270-6474/07/274497-02\$15.00/0 nance imaging (fMRI) scans, Greeble "experts" had higher activity than Greeble novices in the right fusiform face area (rFFA) and the occipital face area when looking at Greebles. More recently, the dorsolateral prefrontal cortex and the intraparietal sulcus have also been suggested to play a role in visual object expertise (Moore et al., 2006). Together, these findings imply that brain regions involved in categorization and expertise can be recruited in the adult brain with learning of new object classes.

Neurophysiologists have applied similar paradigms in macaque monkeys. For instance, Baker et al. (2002) trained monkeys to respond to certain feature combinations. Single units in the inferotemporal cortex showed enhanced selectivity to learned over unlearned stimuli, specifically better neural discrimination without an increase in neuronal firing rates.

These human fMRI and macaque neurophysiology data appear somewhat discrepant in that training increases blood oxygen level-dependent (BOLD) responses in expertise-related areas whereas single units seem to change their tuning rather than increase overall activity. A recent paper by Op de Beeck et al. (2006) in The Journal of Neuroscience may shed some light on this issue. The authors created three novel object classes, which they called "cubies," "smoothies," and "spikies," according to their respective shapes [Op de Beeck et al. (2006), their Fig. 1 (http://www.jneurosci.org/cgi/content/
full/26/50/13025/F1)]. The objects could be varied parametrically, and subjects were trained to discriminate between similar forms within one of the classes. Thus, with nine subjects, three spikies experts, three cubies experts, and three smoothies experts were established. The subjects improved their perceptual skills over $10 \mathrm{~h}$ of training and discriminated the visual objects better with time [Op de Beeck et al. (2006), their Fig. 2 (http://www.jneurosci. org/cgi/content/full/26/50/13025/F2)] . Using high-resolution fMRI with a parallel acquisition technique, subjects were scanned before and after training while watching all three object classes (cubies, smoothies, and spikies). Based on a previously developed approach (Haxby et al., 2001), the authors assumed the following: given that the same objects should activate the same voxels, one might expect a correlation between the BOLD activities evoked by the same stimuli on different days. For instance, voxels strongly activated when the subject saw a spikie should be strongly activated each time the subject is presented with a spikie. This correlation, especially for the untrained stimuli, is an important control for whether two scanning sessions are comparable at all (i.e., whether the scans are aligned spatially or whether the visual cortex responds in a somewhat unvarying manner). In fact, for the two untrained stimulus classes, the responses correlated highly enough that, by resorting to the correlation coefficients, it was possible in 
most cases to decipher the shown stimuli in the BOLD signal of the second scan based on the activity in the first scan.

Given the BOLD increase reported previously for trained stimuli in expertiserelated areas, the authors considered different possible hypotheses as shown in Figure 1 [Op de Beeck et al. (2006), their Fig. 3 (http://www.jneurosci.org/cgi/ content/full/26/50/13025/F3)]. On the one hand, previously active voxels might increase their activity because neurons in an area activated by an untrained stimulus category might respond all the more after training with that particular category. This hypothesis predicts that the voxel activity of the first scan would highly correlate with the second scan, along with a topographically related enhancement. On the other hand, "rewiring" of objectrelated areas might result in a rather unrelated increase in activity. This second hypothesis predicts that the topographical pattern of activity for trained stimuli would be less correlated than for untrained stimuli.

When objects were trained in between the two scans (i.e., when subjects became "object experts"), attempts to classify the second scan based on the correlation to the first scan were much less successful than with the untrained categories [Op de Beeck et al. (2006), their Fig. 6 (http:// www.jneurosci.org/cgi/content/full/26/ 50/13025/F6)]. This decreased correlation speaks in favor of a reorganization of object related areas rather than a mere intensity enhancement of previously activated voxels. Interestingly, the authors found BOLD increases in the lateral occipital complex (LOC) [Op de Beeck et al. (2006), their Fig. 5 (http://www.jneurosci. org/cgi/content/full/26/50/13025/F5)] rather than in the rFFA, the latter of which might have been expected based on work by Gauthier et al. (1999). The LOC plays an important role in object recognition (Grill-Spector et al., 2001). Why some object or shape learning paradigms lead to a BOLD increase in the rFFA and others in the LOC remains an interesting question for future research.

The advance of the study by Op de Beeck et al. (2006) over previous work is the use of high resolution fMRI, combined with the direct comparison of activation patterns measured in the same subjects several weeks later. Their results may help to reconcile the existing discrepancy between previous single-unit and imaging

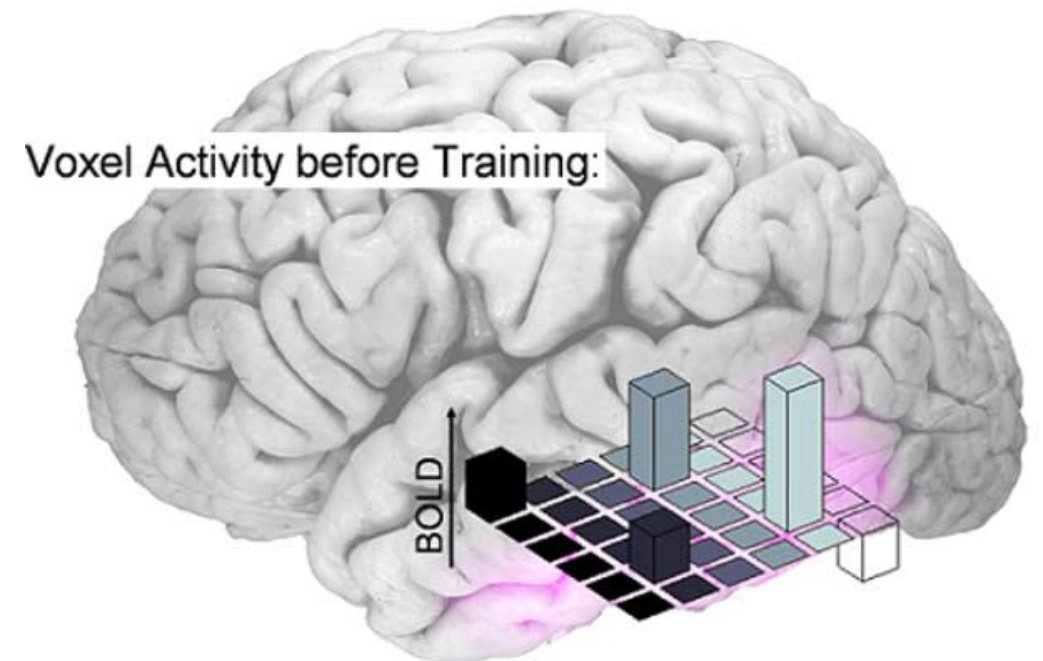

\section{Voxel Activity after Training:}

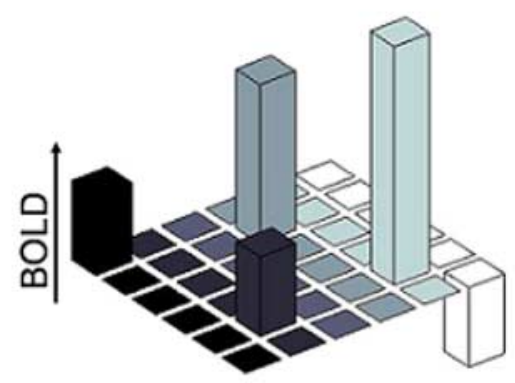

Hypothesis 1

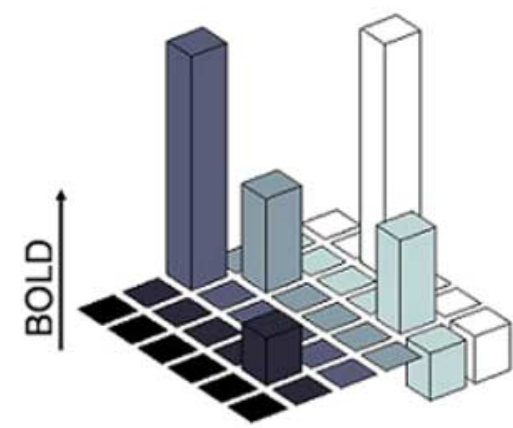

Hypothesis 2

Figure 1. The BOLD signal increases after training in some expertise-related areas. These regions are found in the ventral stream spanning coarsely across the colored patch. Several imaginary voxels are schematically depicted in the $x-y$ plane, with their signal strength to a specific object class indicated on the $z$-axis. Individual voxels activated in the pretraining fMRI session might be activated more strongly in the posttraining scan, as depicted in hypothesis 1. Clearly, pretraining activity and posttraining activity would correlate strongly in this case. Alternatively, one might assume a reorganization of the topographical activation. This second hypothesis implies a decrease in the correlation between pretraining and posttraining scans. Using highresolution fMRI, Op de Beeck et al. (2006) provide evidence in favor of hypothesis 2.

studies. Specifically, the spatially heterogeneous effects of learning observable at finer imaging scales may reflect modifications of neural tuning that are undetectable with larger voxels. It is likely that future technological refinements will bring the spatial scales of neurophysiology and imaging yet closer together and thereby shed even greater light on the neural mechanisms that permit both human and nonhuman primates the remarkable capacity to learn and understand new visual patterns and objects.

\section{References}

Baker CI, Behrman M, Olson CR (2002) Impact of learning on representation of parts and wholes in monkey inferotemporal cortex. Nat Neurosci 5:1210-1216.

Gauthier I, Tarr MJ, Anderson AW, Skudlarski P, Gore JG (1999) Activation of the middle fusiform "face area" increases with expertise in recognizing novel objects. Nat Neurosci 2:568-573.

Grill-Spector K, Kourtzi Z, Kanwisher NG (2001) The lateral occipital complex and its role in object recognition. Vision Res 41:1409-1422.

Haxby JV, Gobbini MI, Furey ML, Ishai A, Schouten JL, Pietrini P (2001) Distributed and overlapping representation of faces and objects in ventral temporal cortex. Science 293:2425-2430.

Logothetis NK, Sheinberg DL (1996) Visual object recognition. Annu Rev Neurosci 19:577-621.

Moore CD, Cohen MX, Ranganath C (2006) Neural mechanisms of expert skills in visual working memory. J Neurosci 26:11187-11196.

Op de Beeck HP, Baker CI, DiCarlo JJ, Kanwisher NG (2006) Discrimination training alters object representations in human extrastriate cortex. J Neurosci 26:13025-13036. 